\title{
Understanding HIV Risk Behavior from a Sociocultural Perspective
}

\section{Claudia E. Ordóñez ${ }^{1}$ and Vincent C. Marconi ${ }^{2,3,4 *}$}

${ }^{1}$ McCord Hospital, Durban, South Africa

${ }^{2}$ Department of Global Health, Rollins School of Public Health, Emory University, Atlanta, USA

${ }^{3}$ Division of Infectious Diseases, Department of Medicine, Emory University School of Medicine, Atlanta, USA

${ }^{4}$ Atlanta Veterans Affairs Medical Center, Decatur, USA

\section{Introduction}

Although the incidence rate for HIV infection varies by population and geography, global estimates have shown a modest decline over the past several years [1]. Accordingly, this past July, the XIX International AIDS Conference that took place in Washington DC adopted the slogan "Turning the Tide Together", signifying that a concerted effort has begun to end the epidemic. Nonetheless, it is important to highlight that within certain communities, these rates have actually increased.

Recently social and cultural aspects such as ethnicity, poverty, gender relations, and geographic region have also been identified as risk factors and have fueled the epidemic among vulnerable populations around the globe $[2,3]$. The 2011 UNAIDS report on the last 30 years of the epidemic warns about today's complex and varied picture at the regional level, with HIV incidence steadily increasing in the Middle East and North Africa and a reversal of the decline in new infections since 2005 for Eastern Europe and Central Asia. The report also highlights that for every three people who start treatment, another five are infected in parts of East and Southern Africa. This information corroborates the considerable variation in HIV prevalence and epidemiological patterns within countries, and show the existence of "epidemic hotspots" [4]

Hotspots exist among men who have sex with men (MSM), ethnic minorities, young women, injection drug users (IDUs) and population groups historically subject to significant inequality. Within a highincome country, the Southern United States has become another example of an epidemic hotspot. Over the course of the HIV epidemic in the U.S., the rate of new infections declined in the early 1990s primarily amongst white MSM but began to rise amongst minority populations for both MSM and heterosexual men and women in urban areas and in the rural south. During this time, poverty, lack of education, injection drug use (IDU) and commercial sex were identified as risk behaviors linked with horizontal HIV transmission [5]. Among low to middle-income countries, South Africa is a well-known example of hyperendemic HIV rates. In these contexts, using a sociocultural ${ }^{1}$ perspective is key to understanding and addressing epidemic hotspots around the world and any associated HIV risk behavior.

\section{Understanding the Individual: The Risk Behavior Paradigm}

According to a classical model of human behavior and HIV risk, individuals are subjected to specific life experiences and, depending upon their underlying personality, can make choices that may put them at risk for HIV acquisition [6]. Further modifying factors include the concomitant presence of substance abuse, depression and other mental

${ }^{1}$ Sociocultural is defined here as the interplay of societal factors (E.g., socia structures, population groups, institutions, and the relationships among them) and cultural factors (E.g., customs, beliefs, values, language.) Thus, the sociocultural perspective refers to social patterns and practices across cultures, how people live in particular places and how they organize, govern, and create meaning. This perspective pays particular attention to ethnicity, sexuality, class, gender, and nationality (adapted from the American Anthropological Association's website). health disorders. Often the choices made can result in additional harmful life experiences leading to new risky behaviors (Figure 1). With time, these patterned responses can increase the likelihood that an individual will establish maladaptive behaviors and addictions; eventually the social life of the individual can destabilize and fracture.

A critical component of this paradigm is the personality. Although debate has surrounded exactly which factors influence personality development, it is generally accepted that both the environment and genetics play a role in this process. Environmental factors especially during childhood and early adolescence that appear to be the most critical include home/family influences, education, socioeconomic status, and the surrounding community. After adolescence, an individual's personality is relatively fixed. Two individuals can respond quite differently to the same intense life experience based upon their personality. An extroverted, risk-taking perspective might satisfy immediate needs (present-biased preference) despite future adverse consequences, whereas an introverted, risk-averse perspective might delay immediate gratification in favor of future benefits. The concept of human agency ${ }^{2}$ adds to the complexity of this model by underscoring the capacity an individual has to challenge and change adverse circumstances and her/his own personality, thus making unexpected choices in terms of risk behavior.

\section{Sociocultural Influences on Behavior}

"AIDS is also a disease lodged in behavioural patterns and value systems that become adapted to the presence of the disease. The people performing these shifts of conduct are not as helpless and passive as our reductionism would have us believe" [7]. The fact that humans are both individual and cultural beings introduces great complexity and variability to understanding risk behaviors. Thus, the sociocultural context can impact an individual's behavior at several access points along the causal pathway previously described. Most directly, the sociocultural milieu provides various scenarios and opportunities that can either trigger maladaptive behavior or can create stressful situations that increase the likelihood risky behavior is undertaken. Furthermore,

${ }^{2}$ Human Agency is the capacity human beings have to make choices and enact them on the world. This concept is defined in contrast to social and cultural factors that seem to limit or influence the opportunities that individuals have.

*Corresponding author: Vincent C. Marconi, The Infectious Disease Program Grady Health System, 341 Ponce de Leon Avenue, Atlanta, GA 30308, USA, E-mail: vcmarco@emory.edu

Received August 01, 2012; Accepted August 02, 2012; Published August 04 2012

Citation: Ordóñez CE, Marconi VC (2012) Understanding HIV Risk Behavio from a Sociocultural Perspective. J AIDS Clinic Res 3:e108. doi:10.4172/2155 $6113.1000 \mathrm{e} 108$

Copyright: (c) 2012 Ordóñez CE, et al. This is an open-access article distributed under the terms of the Creative Commons Attribution License, which permits unrestricted use, distribution, and reproduction in any medium, provided the original author and source are credited. 


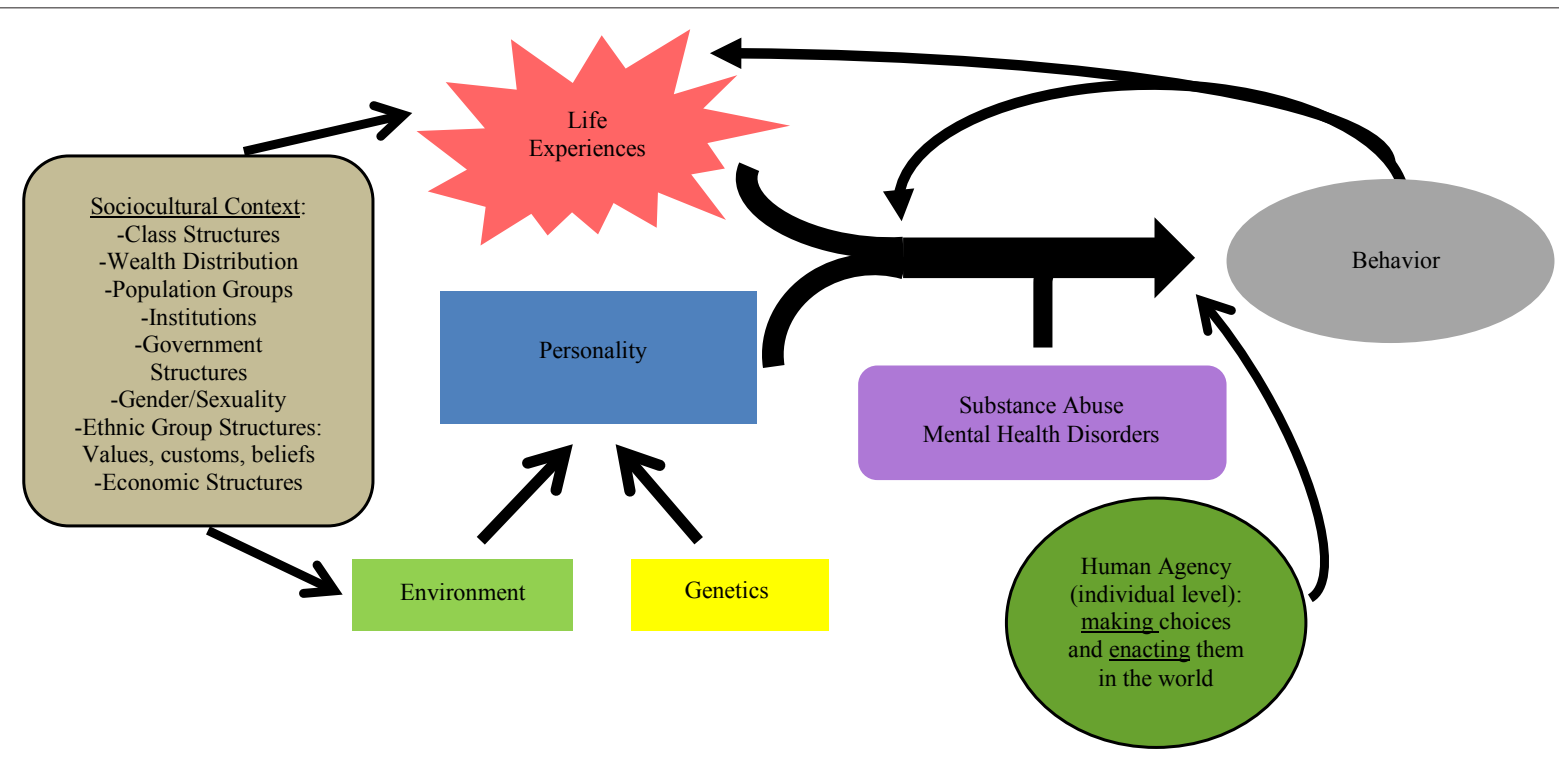

Figure 1: Inclusive Model of Human Behavior. Environment refers to childhood and early adolescent influences on personality development which include the family, education, community, and socioeconomic conditions.

disruption of the cultural context or cultural identity can compromise favorable decision-making. Additionally, as stated above, human agency can also change individual circumstances and even social structures when agency is enacted by several individuals.

Directly connecting with human agency, the concept of social capital $^{3}$ has shown how positive community influences grounded in shared cultural identity can empower individuals to change behavior (by resisting harmful choices) [8] and overcome structural violence ${ }^{4}$. An excellent example of the interaction of social capital and human agency in the context of the HIV epidemic is the behavioral change that happened during the 1980s in the tightly knit, US and European middle-class MSM communities with very intensive support networks and intensive inputs that resulted in effective community-based prevention strategies $[7,9]$.

The clear influence of society and culture in an individual's health risk behaviors makes a strong case for improving the traditionally weak (and often absent) relationship between biomedical and sociocultural paradigms. Effective action to radically change the direction of the HIV epidemic among populations with an increasing incidence rate has been hindered by an incomplete understanding of the cultural, social and political contexts within which it exists and how these connect with the biomedical and psychological realms.

\section{An example: Factors Influencing Risk of HIV Transmission in KwaZulu-Natal, South Africa}

In the case of South Africa and the province of KwaZulu-Natal (KZN), where the HIV prevalence is currently estimated around $40 \%$, the majority of HIV infections are among black South Africans (mostly of Zulu ethnicity) and the likelihood of transmission is overwhelming for sexually active individuals, both the sociocultural and biomedical understandings of the epidemic are key to successfully curbing the epidemic.

Referring to the HIV epidemic in South Africa, Marais emphasizes:

${ }^{3}$ Social capital has been defined slightly different by the various social sciences; however, most perspectives share the core idea that social networks have value In this case, we are referring to the value that social networks can have for people who are living with HIV and members of vulnerable populations.
"Contextualising the disease and linking it to socio-economic and sociocultural dynamics like poverty, migrant labour, income inequalities, financial insecurity, and gender relations are crucial platforms of understanding from which an effective response can be mounted" [7]. Although numerous, high-quality research studies on the South African HIV epidemic have been generated from the social sciences since the 1990s, the tension and insufficient collaboration between these and the biomedical sciences has yet to be overcome.

In discussing the dialectics of the HIV epidemic in Central and East Africa, Friedman and Rossi highlight that as a consequence of colonialism and capitalist processes "...prostitution and unsafe medical injection [syringes shared for medical purposes] became widespread; patterns of concurrent sexual relationships developed or were maintained out of the interaction between economic needs, a mixture of traditional cultural patterns, and their interplay in genderrelated and other politics"[10]. Understanding the effect of recent South African history in the present day is crucial for contextualizing the HIV epidemic. Differing colonial rules (Dutch and English) and the apartheid regime led to lasting and deepening poverty, inequality and underfunded medical systems among other crucial factors associated with the epidemic. Abuses experienced by the non-white population during the apartheid regime resulted in ethnic discrimination, disrupted communities, customs and settlement patterns, and entrenched poverty [11-15]. Black, Indian and Coloured communities were separated (between and within) and isolated from each other and white populations; in this process of relocation individuals and whole communities lost their land, other property, and familial and social groups. A large number of men were separated from their families and sent to work far away in fields and mines, thus breaking the family structure.

${ }^{4}$ Galtung was the first to define structural violence as the avoidable impairment of human needs, diminishing the degree to which someone is able to meet their needs; this type of violence is built into social structures and shows up as unequal power [29]. "The evolving body of work on structural factors that shape vulnerability has made it possible to rethink the nature of discrimination in relation to health The structural violence perspective allows us to re-conceptualize discrimination not merely as an outcome in terms of psychological attitudes of a stigma or prejudice, but as the product of fundamentally unequal social and economic structures..." [29] 
In looking at the contemporary context, numerous studies have explored why this particular region of the world has such a large proportion of individuals living with HIV. Political factors such as HIV denialism (1990s, early 2000s) certainly played a role in the delayed national response to the epidemic $[16,17]$ yet there are many other factors that have also been instrumental in the spread of HIV in this region. Extensive research has examined the influence of individual movement due to migratory labor $[11-13,18,19]$, the commercial sex trade along major highway trucking routes, gender violence/ inequality, and the prevalence of multiple concurrent partnerships [20-26]. Using Friedman and Rossi's [10] analytical concepts, the interaction of economic needs coming from high unemployment and underemployment with cultural patterns (new and old) and their interplay with new social, political, institutional and economic structures have resulted in risky behavioral patterns favoring the growth of the HIV epidemic in KZN and South Africa.

Significant stigma has become a barrier for individuals to disclose their status to their partners or seek care and treatment when treatment for HIV has been shown to be very effective in preventing the spread of infection [27]. Additionally, among many populations, the individual desire and family/community pressure to produce offspring can result in a lowered incentive for condom use. These factors are also clearly associated with depression which has been shown to increase an individual's tendency for risky behavior [28]. We posit that the intersection of Western and Traditional African cultures resulted in a transformed Zulu identity that has been fractured and challenged by structural violence, situating Zulus in an extremely vulnerable position.

\section{Conclusion}

The spread of HIV can be reduced by the application of antiretrovirals universally for those individuals living with HIV and by the use of pre-exposure prophylaxis, condoms and circumcision for those individuals at risk for infection; however, reducing behaviors associated with transmission of HIV will remain a critical component for any prevention strategy in order to "turn the tide". Unfortunately, many of the root causes of HIV transmission are quite complex and systemic in certain regions and require major structural improvements to affect change. These causes include poverty, discrimination, stigma, inadequate education, gender inequality and challenges to cultural identity all of which need further understanding and an effective and creative approach to reduce their impact.

\section{Acknowledgment}

Grant support from Emory University Center for AIDS Research (CFAR) (P30 Al050409) and the Emory School of Medicine Division of Infectious Diseases.

\section{References}

1. UNAIDS (2012) Together we will end AIDS. Geneva, Joint United Nations Programme on HIVIAIDS (UNAIDS)

2. Meditz AL, MaWhinney S, Allshouse A, Feser W, Markowitz M, et al. (2011) Sex, race, and geographic region influence clinical outcomes following primary HIV-1 infection. J Infect Dis 203: 442-451.

3. Beyrer C (2010) Global prevention of HIV infection for neglected populations: men who have sex with men. Clin Infect Dis 3: S108-S113.

4. Joint United Nations Programme on HIVIAIDS (2011) AIDS at 30: Nations at the Crossroads. Geneva: Joint United Nations Programme on HIVIAIDS (UNAIDS).

5. Centers for Disease Control and Prevention (CDC) (2011) Characteristics associated with HIV infection among heterosexuals in urban areas with high AIDS prevalence --- 24 cities, United States, 2006-2007. MMWR Morb Mortal Wkly Rep 60: 1045-1049.

6. Treisman GJ, Angelino AF, Hutton HE (2001) Psychiatric issues in the management of patients with HIV infection. JAMA 286: 2857-2864.
7. Marais H (2000) To the edge: AIDS review 2000.

8. Ware NC, Idoko J, Kaaya S, Biraro IA, Wyatt MA, et al. (2009) Explaining adherence success in sub-Saharan Africa: an ethnographic study. PLoS Med 6: e11.

9. Low-Beer D, Sempala M (2010) Social Capital and Effective HIV Prevention. In Global Health Governance, 4.

10. Friedman SR, Rossi D (2011) Dialectical theory and the study of HIVIAIDS and other epidemics. Dialectical Anthropology 35: 403-427.

11. Collinson MA, Tollman SM, Kahn K (2007) Migration, settlement change and health in post-apartheid South Africa: triangulating health and demographic surveillance with national census data. Scand J Public Health Suppl 69: 77-84.

12. Vearey $J$ (2011) Challenging urban health: towards an improved local government response to migration, informal settlements, and HIV in Johannesburg, South Africa. Glob Health Action

13. Vearey $\mathrm{J}$ (2012) Learning from HIV: exploring migration and health in South Africa. Glob Public Health 7: 58-70.

14. Heeren GA, Jemmott JB 3rd, Tyler JC, Tshabe S, Ngwane Z (2011) Cattle for Wives and Extramarital Trysts for Husbands? Lobola, Men, and HIVISTD Risk Behavior in Southern Africa. J Hum Behav Soc Environ 21: 73-81.

15. Leclerc-Madlala S (2001) Virginity testing: managing sexuality in a maturing HIVIAIDS epidemic. Med Anthropol Q 15: 533-552.

16. Abdool Karim SS, Churchyard GJ, Abdool Karim Q, Lawn SD (2009) HIV infection and tuberculosis in South Africa: an urgent need to escalate the public health response. Lancet 374: 921-933.

17. Doshi RK, del Rio C, Marconi VC (2011) Social Determinants of HIV Health Care: A Tale of Two Cities. In: Understanding HIVIAIDS Management and Care - Pandemic Approaches in the 21st Century. Edited by Kasenga FH: InTech $1-26$.

18. Collinson MA (2010) Striving against adversity: the dynamics of migration, health and poverty in rural South Africa. Glob Health Action 3.

19. Vearey J, Palmary I, Thomas L, Nunez L, Drimie S (2010) Urban health in Johannesburg: the importance of place in understanding intra-urban inequalities in a context of migration and HIV. Health Place 16: 694-702.

20. Reza A, Breiding MJ, Gulaid J, Mercy JA, Blanton C, et al. (2009) Sexual violence and its health consequences for female children in Swaziland: a cluster survey study. Lancet 373: 1966-1972.

21. Hunter M (2005) Cultural politics and masculinities: multiple-partners in historical perspective in KwaZulu-Natal. Cult Health Sex 7: 209-223.

22. Hunter M (2005) Cultural politics and masculinities: Multiple-partners in historical perspective in KwaZulu-Natal. Cult Health Sex 7: 389-403.

23. Jewkes R, Nduna M, Jama Shai N, Dunkle K (2012) Prospective study of rape perpetration by young South african men: incidence \& risk factors. PLoS One 7: e38210.

24. Jewkes R, Morrell R, Sikweyiya Y, Dunkle K, Penn-Kekana L (2012) Transactional relationships and sex with a woman in prostitution: prevalence and patterns in a representative sample of South African men. BMC Public Health 12: 325.

25. Jewkes R, Sikweyiya Y, Morrell R, Dunkle K (2011) Gender inequitable masculinity and sexual entitlement in rape perpetration South Africa: findings of a cross-sectional study. PLoS One 6: e29590.

26. Jewkes R, Sikweyiya Y, Morrell R, Dunkle K (2011) The relationship between intimate partner violence, rape and HIV amongst South African men: a crosssectional study. PLoS One 6: e24256.

27. Cohen MS, Chen YQ, McCauley M, Gamble T, Hosseinipour MC, et al. (2011) Prevention of HIV-1 infection with early antiretroviral therapy. N Engl J Med 365: 493-505.

28. Ramirez-Avila L, Regan S, Giddy J, Chetty S, Ross D, et al. (2012) Depressive Symptoms and Their Impact on Health-seeking Behaviors in Newly-diagnosed HIV-infected Patients in Durban, South Africa. AIDS Behav.

29. Parker R (2012) Stigma, prejudice and discrimination in global public health Cad. Saúde Pública 28: 164-169. 